\title{
THE DISTRIBUTION OF QUADRATIC FUNCTIONALS*
}

\section{By JOHN B. THOMAS (Princeton University) AND EUGENE WONG (IBM Research Center)}

1. Introduction. The purpose of this note is to consider the joint probability distribution of quadratic functionals of the form

$$
y_{m}=\int_{0}^{t} \sum_{j, k} x_{i}(\tau) h_{j k}^{(m)}(\tau) x_{k}(\tau) d \tau
$$

where the $x_{j}(\tau)$ are correlated Gaussian processes and the $h_{j k}^{(m)}(\tau)$ are given functions. The probability distribution of a single functional of the form of (1) has been treated by another method Siegert [1] for the case where the $x_{i}(\tau)$ are stationary.

The solution to the problem described reduces to the solution of a homogeneous matrix integral equation. This equation shows explicitly the transformation functions $h_{i k}^{(m)}$ and the covariance functions of the $x_{i}(\tau)$.

2. Analysis. Let the functionals under consideration be denoted by

$$
y_{m}=\int_{0}^{t} X(\tau) \cdot h^{(m)}(\tau) X(\tau) d \tau, \quad m=1,2, \cdots, p .
$$

Here $X(\tau)$ is an $n$-element column vector whose elements $x_{i}$ are correlated Gaussian processes with zero means, and $h^{(m)}(\tau)$ is an $n \times n$ symmetric matrix [2] whose elements $h_{i k}^{(m)}$ are given functions. In addition let $X(\tau)$ be written formally as the series

$$
X(\tau)=\sum_{k=1}^{\infty} a_{k} \phi_{k}(\tau),
$$

where the $a_{k}$ are random variables and the $\phi_{k}(\tau)$ are non-random vector functions. With the substitution of this series, (2) becomes

$$
y_{m}=\sum_{j=1}^{\infty} \sum_{k=1}^{\infty} a_{j} a_{k} \int_{0}^{t} \phi_{i}(\tau) \cdot h^{(m)}(\tau) \phi_{k}(\tau) d \tau .
$$

The characteristic function for the joint probability distribution of the $y_{m}$ is given by

$$
F\left(\eta_{1}, \cdots, \eta_{p}\right) \triangleq E\left\{\exp \sum_{m=1}^{p} i \eta_{m} y_{m}\right\}
$$

where $i=(-1)^{1 / 2}$ and $E\{\}$ denotes the expectation of the bracketed quantity. From (4), the characteristic function becomes

$$
F\left(\eta_{1}, \cdots, \eta_{p}\right)=E\left\{\exp i \sum_{j=1}^{\infty} \sum_{k=1}^{\infty} a_{i} a_{k} \int_{0}^{t} \phi_{j}(\tau) \cdot w(\tau) \phi_{k}(\tau) d \tau\right\},
$$

where the order of summation has been interchanged and the weighting function matrix $w(\tau)$ has been defined by

$$
w(\tau) \triangleq \sum_{j=1}^{p} \eta_{j} h^{(j)}(\tau) .
$$

Let the elements of the covariance function matrix of the $x_{i}(\tau)$ be given by

$$
R_{j k}(\tau, \sigma) \triangleq E\left\{x_{i}(\tau) x_{k}(\sigma)\right\},
$$

${ }^{*}$ Received Aug. 12, 1960. 
and let the $\phi_{m}(\tau)$ be the eigenfunctions of the homogeneous matrix integral equation

$$
\int_{0}^{t} R(\tau, \sigma) w(\sigma) \phi(\sigma) d \sigma=\lambda \phi(\tau) .
$$

Then it is shown in the next section that the $\phi_{m}(\tau)$ are orthonormal with respect to the weighting function $w(\tau)$, i.e.,

$$
\int_{0}^{t} \phi_{k}(\tau) \cdot w(\tau) \phi_{l}(\tau) d \tau=\delta_{k l}
$$

and the $a_{k}$ are uncorrelated; hence the $a_{k}$ are independent Gaussian variables with zero means and variances $\lambda_{k}$. The characteristic function can be written from (6) and (9) as

$$
F\left(\eta_{1}, \cdots, \eta_{p}\right)=E\left\{\exp i \sum_{k=1}^{\infty} a_{k}^{2}\right\}=\prod_{k=1}^{\infty}\left(1-2 i \lambda_{k}\right)^{-1 / 2} .
$$

Equation (11) is the solution to the problem provided that the series representation (3) is valid and that the eigenvalues $\lambda_{k}$ can be found from (9). These points will now be discussed.

3. Series representation. It will be shown that the vector eigenfunctions of (9) which correspond to different eigenvalues are orthogonal with respect to the weighting function matrix $w(\tau)$. This proof parallels the standard proof for the scalar case [3].

For two distinct eigenvalues $\lambda_{k}$ and $\lambda_{i}$, expression (9) can be used to form

$$
\int_{0}^{t} \phi_{k}(\tau) \cdot w(\tau) \phi_{i}(\tau) d \tau=\frac{1}{\lambda_{k}} \int_{0}^{t} \int_{0}^{t} R(\tau, \sigma) w(\sigma) \phi_{k}(\sigma) \cdot w(\tau) \phi_{j}(\tau) d \sigma d \tau,
$$

and

$$
\int_{0}^{t} \phi_{k}(\tau) \cdot w(\tau) \phi_{i}(\tau) d \tau=\frac{1}{\lambda_{i}} \int_{0}^{t} \int_{0}^{t} \phi_{k}(\tau) \cdot w(\tau) R(\tau, \sigma) w(\sigma) \phi_{i}(\sigma) d \tau d \sigma .
$$

The matrix identity

$$
M A \cdot B=A \cdot M_{T} B,
$$

where the subscript $T$ is used to denote the transpose of the $n \times n$ matrix $M$ and where $A$ and $B$ are $n$-element column vectors, may be applied to the right side of (12) to yield

$$
\int_{0}^{t} \phi_{k}(\tau) \cdot w(\tau) \phi_{i}(\tau) d \tau=\frac{1}{\lambda_{k}} \int_{0}^{t} \int_{0}^{t} \phi_{k}(\sigma) \cdot w_{T}(\sigma) R_{T}(\tau, \sigma) w(\tau) \phi_{i}(\tau) d \sigma d \tau .
$$

Now, if $\sigma$ and $\tau$ are interchanged in (14) and if it is noted that $w_{T}(\tau)=w(\tau)$ and that $R_{T}(\sigma, \tau)=R(\tau, \sigma)$, the result is

$$
\int_{0}^{t} \phi_{k}(\tau) \cdot w(\tau) \phi_{i}(\tau) d \tau=\frac{1}{\lambda_{k}} \int_{0}^{t} \int_{0}^{t} \phi_{k}(\tau) \cdot w(\tau) R(\tau, \sigma) w(\sigma) \phi_{j}(\sigma) d \tau d \sigma .
$$

A comparison of (13) and (15) shows that, for $\lambda_{i} \neq \lambda_{k}$,

$$
\int_{0}^{t} \phi_{k}(\tau) \cdot w(\tau) \phi_{i}(\tau) d \tau=0
$$

and, with proper normalization, (10) follows. 
That the $a_{k}$ of (3) are uncorrelated as a consequence of (9) may be shown by forming $E\left\{a_{k} a_{j}\right\}$. From (10),

$$
E\left\{a_{k} a_{i}\right\}=E\left\{\int_{0}^{t} \int_{0}^{t}\left[X(\tau) \cdot w(\tau) \phi_{k}(\tau)\right]\left[X(\sigma) \cdot w(\sigma) \phi_{i}(\sigma)\right] d \tau d \sigma\right\} .
$$

The integrand of (17) may be rearranged to yield

$$
E\left\{a_{k} a_{j}\right\}=\int_{0}^{t}\left[\int_{0}^{t} E\left\{X(\tau) X_{T}(\sigma)\right\} w(\sigma) \phi_{i}(\sigma) d \sigma\right] \cdot w(\tau) \phi_{k}(\tau) d \tau .
$$

Since $E\left\{X(\tau) X_{T}(\sigma)\right\}=R(\tau, \sigma)$, it follows from (9) that

$$
E\left\{a_{k} a_{j}\right\}=\lambda_{i} \int_{0}^{t} \phi_{i}(\tau) \cdot w(\tau) \phi_{k}(\tau) d \tau,
$$

and, from (10), that

$$
E\left\{a_{k} a_{j}\right\}=\lambda_{i} \delta_{i k} .
$$

Thus the $a_{k}$ are uncorrelated random variables with variances $\lambda_{k}$. The means of the $a_{k}$ are each zero, i.e.

$$
E\left\{a_{k}\right\}=\int_{0}^{t} E\{X(\tau)\} \cdot w(\tau) \phi_{k}(\tau) d \tau=0,
$$

and the linearity of (10) makes the $a_{k}$ Gaussian.

The series expansion (3) is a generalization of the Karhunen-Loeve representation $[4,5]$ and reduces to that representation for the case of one-dimension and for $w(x) \equiv 1$. As in the one-dimensional case, it can be shown $[6,7]$ that the $\phi_{j}$ exist and that the components of the vector series (3) converge mean square to the components of the vector process $X(\tau)$. In addition, if $R(\tau, \sigma)$ is a positive definite kernel, then the $\phi_{i}$ are complete and the $\lambda_{j}$ have at most a finite degeneracy. In these proofs a generalization of Mercer's theorem is required to represent the elements of the matrix $R(\tau, \sigma)$.

4. The single-functional case. For the special case where only a single functional $y$ is considered, (9) can be written as

$$
\int_{0}^{t} R(\tau, \sigma) h(\sigma) \phi(\sigma) d \sigma=\nu \phi(\tau),
$$

and the characteristic function (11) becomes

$$
F(\eta)=\prod_{k=1}^{\infty}\left(1-2 i \eta \nu_{k}\right)^{-1 / 2},
$$

where $\eta \nu_{k}=\lambda_{k}$. Now if the matrix $g(\tau, \mu ; \eta)$ is defined by

$$
g_{1 \mathrm{~m}}(\tau, \mu ; \eta) \triangleq \sum_{k=1}^{\infty} \frac{\nu_{k} \phi_{k}^{(l)}(\tau) \phi_{k}^{(m)}(\mu)}{1-2 i \eta \nu_{k}}
$$

then, by direct evaluation,

$$
g(\tau, \mu ; \eta)-2 i \eta \int_{0}^{t} R(\tau, \sigma) h(\sigma) g(\sigma, \mu ; \eta) d \sigma=R(\tau, \mu) .
$$


For the stationary case (25) is identical, except for a change in notation, with Eq. (79) of Ref. [1]. In terms of the matrix $g(\tau, \mu ; \eta)$ the characteristic function can be written

$$
F(\eta)=\exp i \int_{0}^{\eta} d \xi \int_{0}^{t} \operatorname{Tr}[g(\sigma, \sigma ; \xi) h(\sigma)] d \sigma,
$$

where $\operatorname{Tr}[]$ is the trace of the matrix.

\section{REFERENCES}

1. A. J. F. Siegert, $A$ systematic approach to a class of problems in the theory of noise and other random phenomena-Part III, IRE Transactions on Information Theory IT-4, 4-14 (March 1958)

2. The symmetry of (1) allows $h^{(m)}(\tau)$ to be symmetric without loss of generality

3. Whittaker and Watson, Modern analysis, Cambridge Univ. Press, 1927, Sec. 11.61

4. K. Karhunen, Über lineare Methoden in der Wahrscheinlichkeitsrechnung, Ann. Acad. Sci. Fennicae Ser. A I. Math. Phys., 37 (1947)

5. M. Loeve, Probability theory, D. Van Nostrand Co., Inc., 1955

6. E. Wong, Vector stochastic processes in problems of communication theory, Ph.D. dissertation, Princeton University, May 1959

7. J. K. Wolf, On the detection and estimation problem for multiple nonstationary random processes, Ph.D. dissertation, Princeton University, October 1959

\section{FURTHER EXTENSIONS OF SCHUSTER'S INTEGRAL*}

By E. T. KORNHAUSER** (H. H. Wills Physics Laboratory, University of Bristol)

The integral,

$$
I=\int_{0}^{\infty}\left[C^{2}(x)+S^{2}(x)\right] d x,
$$

where $C(x)$ and $S(x)$ are Fresnel integrals defined by

$$
\begin{aligned}
& C(x)=\int_{x}^{\infty} \cos t^{2} d t, \\
& S(x)=\int_{x}^{\infty} \sin t^{2} d t,
\end{aligned}
$$

was conjectured by Schuster ${ }^{1}$ to have the value $(\pi / 8)^{1}$. Proof that $I$ does in fact have this value was given by Hardy ${ }^{2}$ and more elegantly by Ingham $^{3}$. More recently Bateman ${ }^{4}$ has extended Ingham's treatment to evaluate integrals of the form

$$
\int_{0}^{\infty} C(x) C(a x) d x, \quad \int_{0}^{\infty} C(x) S(a x) d x,
$$

*Received September 1, 1960.

** On leave from Brown University.

'Sir A. Schuster, Proc. Roy. Soc. 107, 15 (1925).

${ }^{2}$ G. H. Hardy, Proc. London Math. Soc. 24, xxx (1926)

${ }^{3}$ A. E. Ingham, J. London Math. Soc. 1, 34 (1926).

'H. Bateman, Proc. Natl. Acad. Sci. 32, 70 (1946).

'C. M. Sparrow, Astrophys. J. 49, 65 (1919). 